PHYSICAL REVIEW E 95, 069903(E) (2017)

\title{
Publisher's Note: Fluctuating lattice Boltzmann method for the diffusion equation [Phys. Rev. E 94, 033302 (2016)]
}

\author{
Alexander J. Wagner and Kyle Strand \\ (Received 14 June 2017; published 22 June 2017)
}

DOI: 10.1103/PhysRevE.95.069903

This paper was published online on 16 September 2016 with errors in Eqs. (58), (59), (62), and (63).

Equation (58) should read as

$$
\partial_{t} \rho+\partial_{\alpha} \eta_{\alpha}+\partial_{t}\left(\tau^{\rho}-\frac{1}{2}\right)\left(\partial_{t} \rho+\partial_{\beta} \eta_{\beta}\right)-\partial_{\alpha}\left(\tau^{j}-\frac{1}{2}\right)\left[\partial_{t} \eta_{\alpha}+\partial_{\beta}\left(\rho \theta \delta_{\alpha \beta}+\chi_{\alpha \beta}\right)\right]=0 .
$$

Equation (59) should read as

$$
\partial_{t} \rho+\partial_{\alpha} \eta_{\alpha}-\partial_{\alpha} \frac{D}{\theta}\left[\partial_{\alpha} \rho \theta+\left(\partial_{t} \eta_{\alpha}+\partial_{\beta} \chi_{\alpha \beta}\right)\right]=0
$$

Equation (62) should read as

$$
\partial_{t} \rho+\partial_{\alpha} \eta_{\alpha}-\partial_{\alpha} \frac{D}{\theta} \partial_{\alpha} \rho \theta=0
$$

Equation (63) should read as

$$
\partial_{t} \rho+\partial_{\alpha} \eta_{\alpha}-\partial_{\alpha} D \partial_{\alpha} \rho=0
$$

The equations have been corrected as of 31 May 2017. The equations are incorrect in the printed version of the journal. 